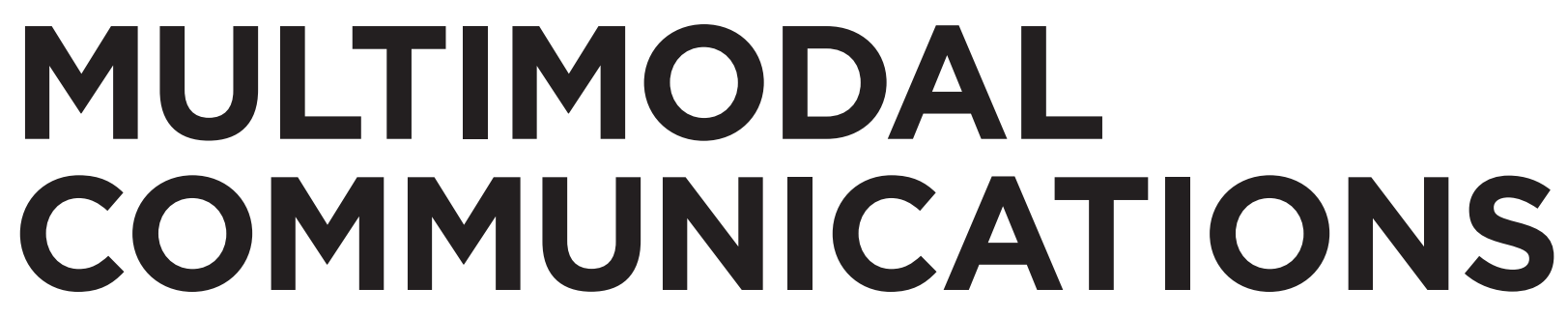

EDITOR IN CHIEF

Sigrid Norris, Auckland

ASSISTANT EDITOR

Jarret Geenen, Nijmegen

\title{
REVIEW EDITOR
}

Jesse Pirini, Wellington

\section{EDITORIAL BOARD}

Elisabeth Adami, Leeds

Najma Al Zidjaly, Muscat

John Bateman, Bremen

Emilia Djonov, Sydney

Gaëlle Ferre, Poitiers

Rodney H. Jones, Hong Kong

Rick Iedema, Sydney

Pia Lane, Oslo

Jay Lemke, San Diego

Carmen Daniela Maier, Aarhus

David McNeill,Chicago

Meredith Marra, Welington

Sarah Pink, Melbourne

Pirkko Raudaskoski, Aalborg

Martin Reisigl, Bern

Jürgen Streeck, Austin

Theo van Leeuwen, Sydney

Karen Wohlwend, Bloomington 
ABSTRACTED/INDEXED IN Baidu Scholar · CNKI Scholar (China National Knowledge Infrastructure) - CNPIEC: cnpLINKer · Dimensions · EBSCO Discovery Service · ERIH PLUS (European Reference Index for the Humanities and Social Sciences) · Google Scholar · IBR (International Bibliography of Reviews of Scholarly Literature in the Humanities and Social Sciences) · IBZ (International Bibliography of Periodical Literature in the Humanities and Social Sciences) · Japan Science and Technology Agency (JST) · J-Gate · JournalTOCs · KESLI-NDSL (Korean National Discovery for Science Leaders) · Microsoft Academic $\cdot$ MLA International Bibliography $\cdot$ MyScienceWork $\cdot$ Naver Academic $\cdot$ Naviga (Softweco) $\cdot$ Norwegian Register for Scientific Journals, Series and Publishers · Primo Central (ExLibris) · Publons · QOAM (Quality Open Access Market) · ReadCube · SCImago (SJR) - SCOPUS · Semantic Scholar · Sherpa/RoMEO · Summon (ProQuest) · TDNet · Ulrich's Periodicals Directory/ulrichsweb · WanFang Data $\cdot$ WorldCat $($ OCLC) $\cdot$ Yewno Discover

e-ISSN 2230-6587

All information regarding notes for contributors, subscriptions, Open access, back volumes and orders is available online at www.degruyter.com/mc

RESPONSIBLE EDITOR Sigrid Norris, Multimodal Research Centre, Auckland University of Technology, New Zealand, multimodalcommunication@outlook.com

COVER IMAGE Lucas Norris

PUBLISHER Walter de Gruyter GmbH, Berlin/Boston, Genthiner Straße 13, 10785 Berlin, Germany

JOURNAL MANAGER Susanne Hoeves, De Gruyter, Genthiner Straße 13, 10785 Berlin, Germany, e-mail: susanne.hoeves@degruyter.com

RESPONSIBLE FOR ADVERTISEMENTS Markus Kügel, De Gruyter, Rosenheimer Str. 143, 81671 München, Germany. Tel.: +49 $8976902-424$, e-mail: anzeigen@degruyter.com.

(C) 2021 Walter de Gruyter GmbH, Berlin/Boston

TYPESETTING TNQ Technologies, Chennai, India 